\title{
Trajectory-Resolved Coulomb Focusing in Tunnel Ionization of Atoms with Intense, Elliptically Polarized Laser Pulses
}

\author{
D. Shafir, ${ }^{1}$ H. Soifer, ${ }^{1}$ C. Vozzi,${ }^{2,3}$ A. S. Johnson, ${ }^{2}$ A. Hartung, ${ }^{2,4}$ Z. Dube, ${ }^{2}$ D. M. Villeneuve, ${ }^{2}$ \\ P. B. Corkum, ${ }^{2}$ N. Dudovich, ${ }^{1}$ and A. Staudte ${ }^{2, *}$ \\ ${ }^{1}$ Department of Physics of Complex Systems, Weizmann Institute of Science, Rehovot 76100, Israel \\ ${ }^{2}$ Joint Attosecond Science Laboratory of the National Research Council and the University of Ottawa, \\ 100 Sussex Drive, Ottawa, Ontario, Canada K1A OR6 \\ ${ }^{3}$ Istituto di Fotonica e Nanotecnologie, CNR, Milan 20133, Italy \\ ${ }^{4}$ Institut für Kernphysik, Goethe Universität, Max-von-Laue Strasse 1, D-60438 Frankfurt, Germany
}

(Received 12 November 2012; published 9 July 2013)

\begin{abstract}
In strong-field light-matter interactions, the strong laser field dominates the dynamics. However, recent experiments indicate that the Coulomb force can play an important role as well. In this Letter, we have studied the photoelectron momentum distributions produced from noble gases in elliptically polarized, $800 \mathrm{~nm}$ laser light. By performing a complete mapping of the three-dimensional electron momentum, we find that Coulomb focusing significantly narrows the lateral momentum spread. We find a surprisingly sensitive dependence of Coulomb focusing on the initial transverse momentum distribution, i.e., the momentum at the moment of birth of the photoelectron. We also observe a strong signature of the low-energy structure in the above threshold ionization spectrum.
\end{abstract}

DOI: 10.1103/PhysRevLett.111.023005

PACS numbers: $32.80 . \mathrm{Rm}, 32.80 . \mathrm{Fb}, 34.80 . \mathrm{Bm}, 34.80 . \mathrm{Qb}$

In traditional collision physics, an electron is propelled at a target and detected after the scattering event. The two important parameters in this process are the electron's energy and impact parameter. Whereas the energy can be tuned very accurately, the impact parameter cannot be controlled. Laser induced recollision with photoelectrons [1] opens up new perspectives in manipulating the collision via the shaping of the laser field. Recollision gives rise to a wealth of phenomena such as laser induced electron diffraction [2], high harmonic orbital tomography [3], and attosecond pulses [4]. Whereas the first order modeling of these phenomena neglects the parent ion [5], evidence is abound of its impact on the recolliding electron (e.g., [6-10]). In particular, the momentum distribution transverse to the polarization plane has been shown to provide a window into the interaction of the photoelectron with its parent ion potential $[6,11]$. However, a complete characterization of the Coulomb effects on the photoelectron momentum requires two things: first, the ability to distinguish between the Coulomb field of the ion and the laser field, and second, the ability to distinguish between different electron trajectories. Whereas the former allows us to quantify the strength of the effect, the latter allows us to differentiate the primary parameters of the recollision, i.e., electron energy at recollision and the minimal distance from the ion.

Here, we show that we can satisfy both requirements by performing a three-dimensional (3D) momentum measurement of photoelectrons from noble-gas atoms produced by elliptically polarized, intense laser fields. We separate different electron trajectories by adding a small ellipticity to the fundamental driving field. We devise a new way to analyze the transverse momentum distribution resolved for electron trajectories. We confirm that Coulomb effects depend on the recollision parameters, which in turn depend sensitively on the initial conditions of the tunneled wave packet. This yields the counterintuitive result that narrower momentum distributions right after tunneling lead to wider asymptotic momenta perpendicular to the tunneling direction. Finally, our approach of trajectory resolved analysis of the transverse momentum naturally lends itself to an explanation of the puzzling so-called low-energy structure (LES) in the photoelectron spectrum [7].

The experiment was performed using cold target recoil ion spectroscopy (COLTRIMS) [12]. The $800 \mathrm{~nm}, 40 \mathrm{fs}$, $3 \mu \mathrm{J}$ laser pulses were supplied by a $100 \mathrm{kHz}$ regenerative Ti:sapphire amplifier and focused by a $f=50 \mathrm{~mm}$ parabolic mirror into a continuous supersonic gas jet. Ions and electrons were guided by homogeneous and parallel electric $(19.5 \mathrm{~V} / \mathrm{cm})$ and magnetic $(12.8 \mathrm{G})$ fields onto position sensitive detectors. Electrons pass through an acceleration region $(63.3 \mathrm{~mm})$ followed by a field-free drift region $(140 \mathrm{~mm})$ before reaching the detector. The momentum resolution for electrons is estimated to be $\Delta p_{x}=\Delta p_{y}<0.01$ a.u. and $\Delta p_{z} \approx 0.1$ a.u. Polarization control was achieved by rotating a $\lambda / 2$ plate in front of a broadband $\lambda / 4$ plate.

In strong-field ionization, the oscillating laser field drives the propagation of the ionized electron. If the electron is ionized before the peak of the electric field within a half optical cycle, it is pulled away from the ion and reaches the detector directly, hence, these electrons are called direct electrons. If the electron ionizes after the peak of the electric field, it is driven back to the parent ion before reaching the detector on the opposite side. These 
electrons are called rescattering electrons. For the following optical half cycle, the situation is reversed. Whereas direct electrons experience a Coulomb potential only right after ionization when escaping the parent ion [11], rescattered electrons in addition will be influenced by the parent ion's potential upon their return $[6,13]$.

Formally, neglecting the ion potential, the final electron momentum distribution is proportional to the vector potential $A$ of the electric field $E$ :

$$
p_{z}^{\mathrm{final}}\left(t_{0}\right) \propto p_{z}\left(t_{0}\right)+A\left(t_{0}\right)=p_{z}\left(t_{0}\right)-\int_{t_{0}}^{\infty} E(t) d t
$$

where $p_{z}\left(t_{0}\right)$ is the initial momentum distribution after the tunneling ionization instant $t_{0}$ and $E(t)=E_{\max } f(t) \sin \omega t$ is the laser pulse of peak field strength $E_{\max }$, envelope $f(t)$, and frequency $\omega$. Equation (1) shows that in linear polarization the final momentum is degenerate, i.e., direct and rescattered electrons associated with the same vector potential $A$ reach the detector with the same final momentum.

A simple way to separate the different trajectories while still allowing for rescattering is to employ slightly elliptically polarized light. Figure 1(a) illustrates electron trajectories in the polarization plane of an elliptically polarized laser pulse (ellipticity $\epsilon=0.3$ ). Shown are projections of the trajectories onto the spatial dimensions coinciding with the polarization components and the time axis. The field component of the minor polarization axis in $y$ separates previously overlapping trajectories of direct and rescattered electrons, indicated in black and orange, respectively. However, the measured momentum distribution, shown in Fig. 1(b), does not reflect the fourfold symmetry of the polarization ellipse. This asymmetry is due the parent ion's Coulomb field. In addition to focusing the transverse momentum, the Coulomb field also distorts the angular distribution in the polarization plane (see, e.g., [14-19]). While the so-called Coulomb asymmetry complicates the separation of trajectories, a measurement along the $x$ axis allows us to isolate the Coulomb effects for different trajectories under field free conditions. For each trajectory, the $p_{x}$ distribution is influenced only by the tunneling process and the parent ion's field.

The three panels on the right in Fig. 1(b) show the $p_{x}$ distribution measured in $\mathrm{Ar}$ for three $\left(p_{y}, p_{z}\right)$, representing distinct trajectories. Panel A represents the direct trajectories. Panel B corresponds to rescattered trajectories, which have been steered by the elliptically polarized field back to the ion with a large impact parameter or high velocity. Panel C selects electrons with zero energy, corresponding to those rescattered trajectories that have a small impact parameter and a low recollision velocity. Comparing these three cuts, we observe a substantial difference. Electrons in panel A are influenced by the Coulomb potential primarily upon leaving the parent ion's vicinity, yielding a largely undistorted Gaussian distribution. In panel B, the ionic potential acts on the electron after tunneling and during
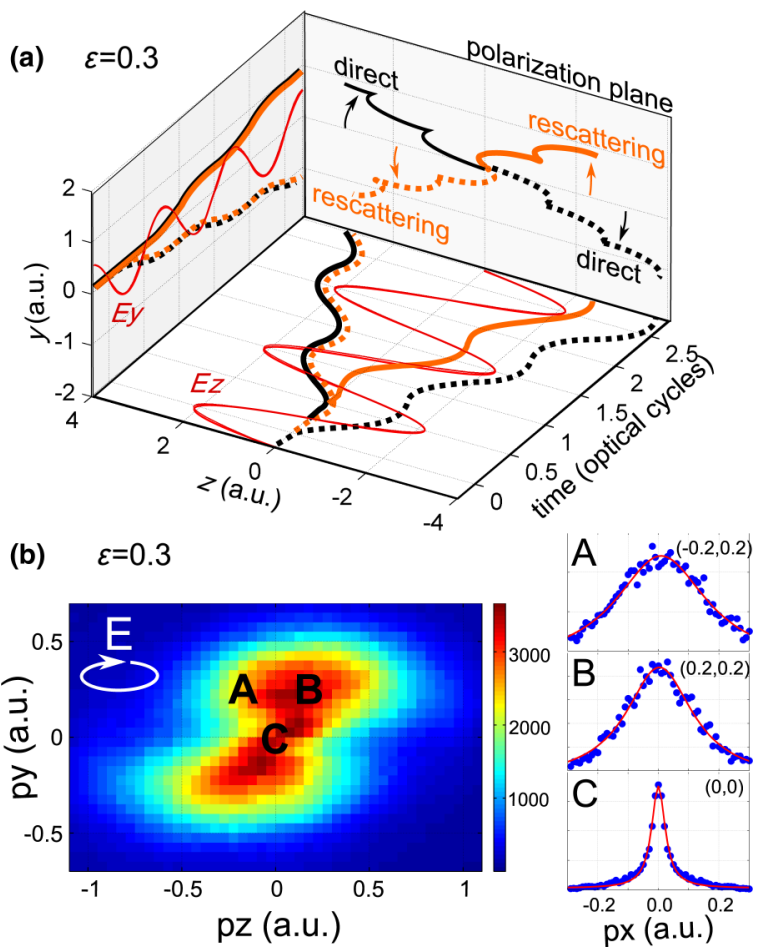

FIG. 1 (color online). (a) Examples of direct and rescattered trajectories in elliptically polarized light in the $y-z$ polarization plane. Black indicates direct, and orange indicates rescattering trajectories. Dotted and solid trajectories indicate ionization in subsequent optical half cycles. Arrows indicate the separation of trajectories due to nonzero ellipticity. (b) Measured photoelectron momentum of $\mathrm{Ar}$ in the $y-z$ polarization plane of elliptically polarized $(\epsilon=0.3)$ laser pulses at a peak intensity of $1.5 \times 10^{14} \mathrm{~W} / \mathrm{cm}^{2}$. Right column: $p_{x}$ momentum at indicated $\left(p_{z}, p_{y}\right)$. Red lines are a fit to the $p_{x}$ distribution using Eq. (2) (a.u. denotes atomic units).

recollision. A significant narrowing of the rescattered electrons can be observed. The very narrow transverse momentum in panel $\mathrm{C}$ reveals a much stronger Coulomb focusing for a low rescattering velocity and small impact parameter.

Hence, Fig. 1(b) shows that Coulomb focusing depends on two parameters - the recollision velocity and the impact parameter. The lower the velocity and the smaller the impact parameter at the time of recollision, the tighter the focusing will be. The ellipticity of the laser field maps the two parameters. The velocity at recollision is mapped onto the momentum component along the major axis, and the impact parameter is mapped onto the minor axis.

To resolve Coulomb effects by trajectory, we need to select an ellipticity $\epsilon=E_{\text {min }} / E_{\text {maj }}$ that is large enough to separate the trajectories from opposite half cycles without suppressing recollision completely [20]. We assume that the ionization proceeds along the major electric field component $E_{\text {maj }}$ parallel to the $z$ axis. The minor electric field component $E_{\min }$ along the $y$ axis then separates direct and rescattered trajectories from opposite half cycles by up to $2 E_{\min } / \omega$. The transverse momentum width after tunneling 
$(1 \sigma)$ is $p_{\perp}^{\mathrm{ADK}}=\left(E_{\text {maj }} / \sqrt{2 V_{\text {ion }}}\right)^{1 / 2}$, where $V_{\text {ion }}$ is the ionization potential [21]. To separate the trajectories by $2 p_{\perp}^{\mathrm{ADK}}$, an ellipticity of $\epsilon \geq \omega E_{\text {maj }}^{-1 / 2}\left(2 V_{\text {ion }}\right)^{-1 / 4}$ is required. For $\mathrm{Kr}\left(V_{\text {ion }}=13.99 \mathrm{eV}\right)$ and $E_{\text {maj }}=0.05$ a.u., this requirement yields a minimum ellipticity of $\epsilon=0.25$. Under these conditions, the center of the recolliding wave packet is displaced from the nucleus by $\Delta y \approx \epsilon E_{\mathrm{maj}} / \omega^{2}=3.8$ a.u., whereas the spatial width of the returning wave packet is $\approx(3 / 4) 2 \pi / \omega p_{\perp}^{\mathrm{ADK}} \approx 18$ a.u.. Figure 2 (a) shows the photoelectron momentum distribution of $\mathrm{Kr}$ in the polarization plane of $40 \mathrm{fs}, 800 \mathrm{~nm}$ laser pulses with $\epsilon=0.3$ and a peak intensity of $(1.5 \pm 0.15) \times 10^{14} \mathrm{~W} / \mathrm{cm}^{2}$.

A complete picture of trajectory-resolved Coulomb focusing is obtained by analyzing the transverse momentum for each point in the polarization plane. Specifically, we fit the transverse momentum distribution with a Lorentz distribution for each combination of $p_{z}$ and $p_{y}$ components

$$
\frac{d N\left(p_{y}, p_{z}\right)}{d p_{x}}=\frac{A}{\pi \gamma\left(1+p_{x}^{2} / \gamma^{2}\right)} .
$$

The fit parameter $\gamma=\gamma\left(p_{y}, p_{z}\right)$ corresponds to the half width at half maximum (HWHM). In Fig. 2(c), we plot the two-dimensional map of the transverse momentum width $\gamma\left(p_{y}, p_{z}\right)$ for $\mathrm{Kr}$ atoms. It allows us to observe how Coulomb focusing depends on the electron trajectory. Areas of predominantly direct electrons exhibit a wider transverse momentum than areas where rescattered
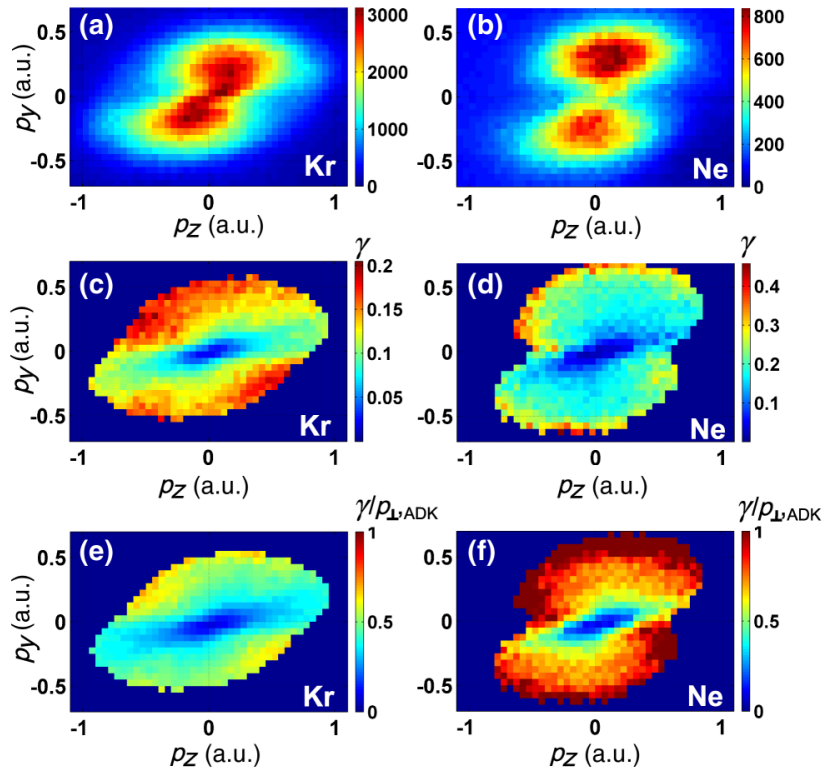

FIG. 2 (color online). Photoelectron momentum distributions of (a) $\mathrm{Kr}$ and (b) $\mathrm{Ne}$ in the polarization plane of elliptically $(\epsilon=0.3)$ polarized light at a peak intensity of $(1.5 \pm 0.15) \times$ $10^{14} \mathrm{~W} / \mathrm{cm}^{2}$ ( $E_{z}=0.05$ a.u. ). (c) and (d) HWHM $\gamma\left(p_{y}, p_{z}\right)$ for each bin in (a) and (b), respectively. (e) and (f) data from (c) and (d) are normalized with the expectation value of tunneling theory. trajectories dominate. Also, pronounced Coulomb focusing [13] for the photoelectrons with the lowest observable momentum in the polarization plane is revealed. The transverse momentum is narrower for events associated with a small $p_{y}$ momentum, i.e., in the direction of the minor polarization axis. These electrons are ionized with an initial momentum along the minor field axis such that upon rescattering, they approach the ion at a low impact parameter and are focused along both the $x$ and the $y$ axis.

A deeper understanding of the interplay between the ion potential and initial transverse momentum distribution is obtained when $p_{\perp}^{\mathrm{ADK}}$ is varied under otherwise identical conditions. This can be achieved by comparing two atomic species. Figure 2(b) shows the photoelectron momentum distribution of Ne under the same conditions as Kr. The photoelectrons associated with $\mathrm{Kr}$ in Fig. 2(a) exhibit a strong Coulomb asymmetry and a distinct peak at zero momentum. In contrast, the photoelectron spectrum associated with $\mathrm{Ne}$ shows a weaker angular distortion and fewer zero energy electrons, suggesting a smaller influence of the Coulomb potential.

For $\mathrm{Ne}$, the initial transverse momentum is expected to be about $10 \%$ narrower than that of $\mathrm{Kr}$ in the conditions of our experiment. Generally speaking, in elliptical polarization, a narrower transverse momentum at the exit of the tunnel results in a smaller overlap of the rescattering wave packet with the parent ion. Figure 2(d) shows that the transverse width of the final momentum is considerably wider for $\mathrm{Ne}$ than $\mathrm{Kr}$. Thus, even marginal differences in the initial conditions can lead to pronounced differences in the final momentum.

In Figs. 2(e) and 2(f), we normalized $\gamma\left(p_{y}, p_{z}\right)$ with the HWHM prediction of tunneling theory, $p_{\perp, \mathrm{HWHM}}^{\mathrm{ADK}}=$ $\sqrt{2 \ln 2} p_{\perp}^{\mathrm{ADK}}$. This allows a direct comparison of both atoms, removing the $V_{\text {ion }}$ dependence of the width $p_{\perp}^{\mathrm{ADK}}$, therefore isolating the influence of the Coulomb field. Here, we used an average normalization, neglecting the dependence of the initial transverse momentum width on the instantaneous field strength [22]. The normalization shows that in Kr, Fig. 2(e), the Coulomb field distorts the momentum space globally, focusing even the direct trajectories down to $1 / 2$ the width of Coulomb-free tunneling theory. In contrast, for $\mathrm{Ne}$ [Fig. 2(f)], tunneling theory describes $\gamma\left(p_{y}, p_{z}\right)$ much better for the direct and even the soft rescattered trajectories. This observation agrees with the lack of zero energy electrons and a smaller Coulomb asymmetry in Ne.

Our analysis directly provides insight into the origin of the so-called low-energy structure (LES). Figure 3(a) shows the photoelectron energy spectra for $\mathrm{Kr}$ (red line) and $\mathrm{Ne}$ (blue line) integrated over all momentum components. The observed low-energy electrons are a manifestation of a puzzling energy structure thought to be exclusive to linearly polarized, midinfrared laser fields [7,23]. Only recently, indications were found of LES at a laser 

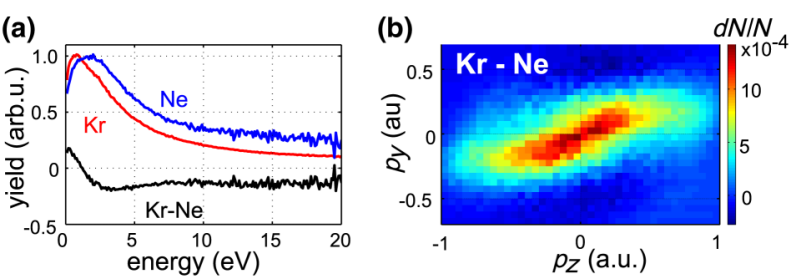

FIG. 3 (color online). Low-energy structure in the photoelectrons (from data in Fig. 2). (a) Photoelectron energy spectrum normalized to peak value for $\mathrm{Kr}$ (red), $\mathrm{Ne}$ (blue), and the difference (black). (b) Difference of normalized momentum distributions in 2(a) and 2(b).

wavelength of $800 \mathrm{~nm}$ [24]. Hence, theoretical efforts concentrated on the midinfrared [25-30]. It was argued that the LES originates in the Coulomb focusing of transverse momentum components [25,26,29]. However, a slowdown, or bunching of longitudinal momenta, has also been proposed as mechanism for LES [28]. The difference of the energy spectra for the two atomic species (black line) in Fig. 3(a) demonstrates that LES depends on the initial momentum distribution. From the difference of the two-dimensional momentum distributions [shown in Fig. 3(b)], we conclude that recollision trajectories contribute primarily to the enhancement of LES. Hence, we validate the hypothesis of transverse momentum focusing of recolliding trajectories. LES depends on the transverse momentum at the exit of the tunnel and LES electrons are not only focused parallel to the major field axis, but also perpendicular to the light field polarization.

We now present a systematic study of Coulomb focusing as a function of ionization potential, intensity, and ellipticity. For this purpose, we extract a weighted average of the fitted transverse momentum width $\hat{p}_{x}=$ $\left(1 / N_{\text {tot }}\right) \sum_{z} \sum_{y} N_{y z} \gamma\left(p_{y}, p_{z}\right)$, where $N_{\text {tot }}$ is the total number of electrons measured and $N_{y z}$ the number of photoelectrons in a given bin in the polarization plane. The weighted average reduces the influence of systematic errors in determining the transverse width and enables a parametrization of the Coulomb effect.

Figure 4(a) presents $\hat{p}_{x}$ for two intensities at an ellipticity of $\epsilon=0.3$ and for different atomic species (Xe, $\mathrm{Kr}, \mathrm{Ar}$, $\mathrm{Ne}$ ). Xe and $\mathrm{Ne}$ could not be measured at the higher and lower intensity, respectively, due to saturation in Xe and too little ionization in Ne. In Fig. 4(b), the average transverse width for a scan of the light ellipticity is shown for $\mathrm{Kr}$ where the field strength of the major axis $E_{\text {maj }}$ was kept constant at 0.041 a.u.. Thus, we vary two parameters: the initial momentum width through variation of intensity and $V_{\text {ion }}$, and the impact parameter at recollision through the variation of the ellipticity.

In order to remove the influence of the $V_{\text {ion }}$ and field dependence on the tunnelled wave packet, we normalize each measured $\hat{p}_{x}$ by its corresponding $p_{\perp, \mathrm{HWHM}}^{\mathrm{ADK}}$ in Figs. 4(c) and 4(d). Thereby, we can compare the
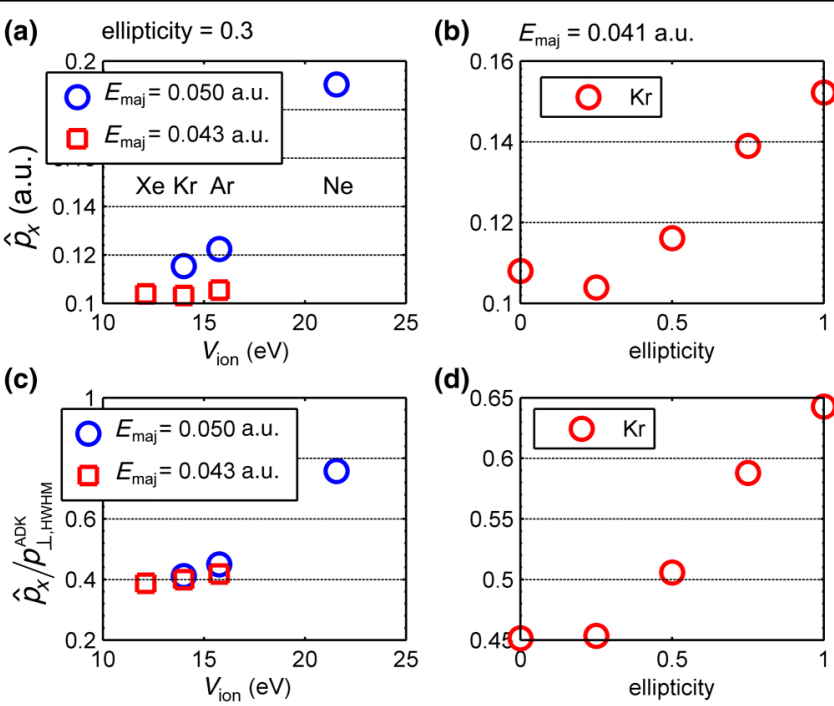

(d)

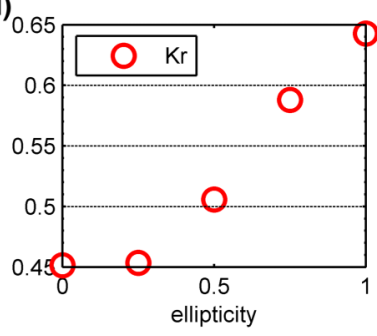

FIG. 4 (color online). Weighted sum of the fitted widths $\gamma\left(p_{y}, p_{z}\right)$ of photoelectron distributions. (a) red squares: $\mathrm{Xe}$, $\mathrm{Kr}$, and Ar at $(1.1 \pm 0.1) \times 10^{14} \mathrm{~W} / \mathrm{cm}^{2}$, blue circles : $\mathrm{Kr}, \mathrm{Ar}$, and $\mathrm{Ne}$ at $(1.5 \pm 0.15) \times 10^{14} \mathrm{~W} / \mathrm{cm}^{2}$. (b) ellipticity scan in $\mathrm{Kr}$ with a constant $E_{\text {maj }}=0.041$ a.u. (c) and (d) ratio of measured width and the prediction of tunneling theory [21] for the corresponding data in (a) and (b).

experiments directly to the Coulomb-free tunneling theory. Contrary to the predicted $V_{\text {ion }}^{-1 / 4}$ dependence of the transverse momentum [21], the observable photoelectron momentum exhibits a monotonic increase of $\hat{p}_{x}$ with $V_{\text {ion }}$. Hence, Coulomb focusing has a tremendous impact on the observable momentum. However, the correlation with the field strength is well captured by the theory [see $\mathrm{Kr}$ and $\mathrm{Ar}$ in Fig. 4(c)]. When the impact parameter for the rescattering electron is changed through an increase in ellipticity, the average transverse momentum approaches the Coulomb-free prediction but consistently remains narrower [Fig. 4(d)].

In conclusion, our study is an important step in understanding the general behavior of long-range potentials and their influence on strong-field light-matter interaction. We have introduced the trajectory-resolved analysis of Coulomb effects on the photoelectron momentum perpendicular to the tunneling direction. We observed the low-energy structure in the photoelectron spectrum of elliptically polarized, $800 \mathrm{~nm}$ light, and we validated the importance of the initial transverse momentum for the appearance of LES. Finally, our data quantifies one aspect of how Coulomb effects will become increasingly important for complex systems with a lower ionization potential -with the width of the initial transverse momentum distribution. This type of trajectory-resolved analysis can be applied, for example to attosecond angular streaking of molecular ionization [18], to characterize the tunneled wave packet [19], and to photoelectron holography [31]. 
We acknowledge the DAAD, the Minerva foundation, the Israeli Science foundation, and the Crown Center of Photonics for financial support. H.S. is supported by the Adams Fellowship Program of the Israel Academy of Sciences and Humanities.

Note added in proof.-In a recent, complementary work on Coulomb asymmetry by Li et al. [32] the quarter cycle separation of trajectories in the polarization plane of elliptically polarized light was verified using a semi-classical simulation.

*andre.staudte@nrc.ca

[1] P. B. Corkum, Phys. Rev. Lett. 71, 1994 (1993).

[2] M. Meckel et al., Science 320, 1478 (2008).

[3] J. Itatani, J. Levesque, D. Zeidler, H. Niikura, H. Pépin, J. C. Kieffer, P. B. Corkum, and D. M. Villeneuve, Nature (London) 432, 867 (2004).

[4] F. Krausz and M. Yu. Ivanov, Rev. Mod. Phys. 81, 163 (2009).

[5] M. Lewenstein, Ph. Balcou, M. Yu. Ivanov, A. L'Huillier, and P. B. Corkum, Phys. Rev. A 49, 2117 (1994).

[6] D. Comtois, D. Zeidler, H. Pépin, J.C. Kieffer, D. M. Villeneuve, and P.B. Corkum, J. Phys. B 38, 1923 (2005).

[7] C. I. Blaga, F. Catoire, P. Colosimo, G. G. Paulus, H. G. Muller, P. Agostini, and L. F. DiMauro, Nat. Phys. 5, 335 (2009).

[8] Y. Liu et al., Phys. Rev. Lett. 104, 173002 (2010).

[9] C. Vozzi, M. Negro, F. Calegari, G. Sansone, M. Nisoli, S. De Silvestri, and S. Stagira, Nat. Phys. 7, 822 (2011).

[10] D. Shafir et al., Phys. Rev. Lett. 108, 203001 (2012).

[11] L. Arissian, C. Smeenk, F. Turner, C. Trallero, A. V. Sokolov, D. M. Villeneuve, A. Staudte, and P. B. Corkum, Phys. Rev. Lett. 105, 133002 (2010).

[12] J. Ullrich, R. Moshammer, A. Dorn, R. Dörner, L. Ph H. Schmidt, and H. Schmidt-Böcking, Rep. Prog. Phys. 66, 1463 (2003).

[13] T. Brabec, M. Yu. Ivanov, and P. B. Corkum, Phys. Rev. A 54, R2551 (1996).
[14] M. Bashkansky, P. H. Bucksbaum, and D. W. Schumacher, Phys. Rev. Lett. 60, 2458 (1988).

[15] S. P. Goreslavski, G. G. Paulus, S. V. Popruzhenko, and N. I. Shvetsov-Shilovski, Phys. Rev. Lett. 93, 233002 (2004).

[16] M. Odenweller, N. Takemoto, A. Vredenborg, K. Cole, K. Pahl, J. Titze, L. Ph. H. Schmidt, T. Jahnke, R. Dörner, and A. Becker, Phys. Rev. Lett. 107, 143004 (2011).

[17] N. I. Shvetsov-Shilovski, D. Dimitrovski, and L. B. Madsen, Phys. Rev. A 85, 023428 (2012).

[18] M. Spanner et al., J. Phys. B 45, 194011 (2012).

[19] A. N. Pfeiffer, C. Cirelli, A. S. Landsman, M. Smolarski, D. Dimitrovski, L. B. Madsen, and U. Keller, Phys. Rev. Lett. 109, 083002 (2012).

[20] G. G. Paulus, F. Zacher, H. Walther, A. Lohr, W. Becker, and M. Kleber, Phys. Rev. Lett. 80, 484 (1998).

[21] N. B. Delone and V. P. Krainov, J. Opt. Soc. Am. B 8, 1207 (1991).

[22] J. Henkel, M. Lein, V. Engel, and I. Dreissigacker, Phys. Rev. A 85, 021402(R) (2012).

[23] W. Quan et al., Phys. Rev. Lett. 103, 093001 (2009).

[24] C. Y. Wu, Y.D. Yang, Y. Q. Liu, Q. H. Gong, M. Wu, X. Liu, X. L. Hao, W. D. Li, X. T. He, and J. Chen, Phys. Rev. Lett. 109, 043001 (2012).

[25] C. Liu and K.Z. Hatsagortsyan, Phys. Rev. Lett. 105, 113003 (2010).

[26] T.-M. Yan, S. V. Popruzhenko, M. J. J. Vrakking, and D. Bauer, Phys. Rev. Lett. 105, 253002 (2010).

[27] D. A. Telnov and Shih-I Chu, Phys. Rev. A 83, 063406 (2011).

[28] A. Kästner, U. Saalmann, and J.-M. Rost, Phys. Rev. Lett. 108, 033201 (2012).

[29] C. Lemell, K. I. Dimitriou, X.-M. Tong, S. Nagele, D. V. Kartashov, J. Burgdörfer, and S. Gräfe, Phys. Rev. A 85, 011403 (2012).

[30] C. Liu and K.Z. Hatsagortsyan, Phys. Rev. A 85, 023413 (2012).

[31] D. D. Hickstein et al., Phys. Rev. Lett. 109, 073004 (2012).

[32] M. Li, Y. Liu, H. Liu, Q. Ning, L. Fu, J. Liu, Y. Deng, C. Wu, L. Peng, and Q. Gong, following paper, Phys. Rev. Lett. 111, 023006 (2013). 